\title{
Rational Identification of Prognostic Markers of Breast Cancer
}

\author{
Maysson Al-Haj Ibrahim ${ }^{1,2}$, Joanne L Selway ${ }^{2}$, Kian Chin $^{3}$, Sabah Jassim¹, \\ Michael A. Cawthorne ${ }^{2}$ and Kenneth Langlands ${ }^{2}$ \\ ${ }^{1}$ Department of Applied Computing, Buckingham University, Buckingham, U.K. \\ ${ }^{2}$ Buckingham Institute for Translational Medicine, Buckingham University, Buckingham, U.K. \\ ${ }^{3}$ Department of Surgery, Milton Keynes Hospital NHS Foundation Trust, Milton Keynes, U.K.
}

Keywords: Disease Classification, Breast Cancer, Prognosis, Biomarkers, Metabolic Networks and Pathways, Gene Regulatory Networks, Microarray Analysis.

\begin{abstract}
Accurate prognostication is central to the management of breast cancer, and traditional clinical and histochemical-based assessments are increasingly augmented by genetic tests. In particular, the use of microarray data has allowed the creation of molecular disease signatures for the early identification of individuals at elevated risk of relapse. However, tailoring therapy on the basis of a molecular assay is only recommended in certain cases, and the identification of a minimal set of genes whose expression allows informed decision-making in a broader spectrum of disease remains challenging. Finding an optimal solution is, however, an intractable computational task (i.e. retrieving the smallest group of genes with the greatest prognostic power). Our solution was to reduce the genetic search-space by using two filtering steps that enriched by biological function those genes whose expression discriminated disease states. In this way, we were able to identify a new molecular signature, the expression characteristics of which facilitated the classification of intermediate risk disease. We went on to create a statistical test that confirmed the relevance of our approach by comparing the performance of our signature to that of 1000 random signatures.
\end{abstract}

\section{INTRODUCTION}

Assessment of a number of clinical features is made at presentation in order to identify women at elevated risk of an aggressive disease course, and so inform disease management. These include estrogen receptor (ER) status, size of the primary lesion and lymph node involvement. Adjuvant hormone therapy is very effective in preventing recurrence of disease, but side effects are such that sparing individuals at low risk of relapse from intensive therapy has significant quality of life implications. However, there is a considerable margin of error, and as many as $80 \%$ of women may be over-treated and improving outcomes by identifying a more informative list of biomarkers remains a challenging task (Van't Veer et al., 2002). Genome-wide transcriptional profiling methods (such as microarrays) provide a snap-shot of gene activity in a cell, and by correlating patterns of gene expression in the primary lesion with outcomes, a number of investigators have sought to identify molecular signatures characteristic of different tumour subtypes (Wesolowski, 2011).

Diagnostic tests informed by molecular signatures of high risk disease are available for use in clinical practice, and recently use of the Oncotype DX Breast Cancer assay (Paik et al., 2004) was approved by the UK's National Institute for Clinical Excellence (NICE) for use in the management of a sub-group of patients with an intermediate risk of tumour recurrence (guidance.nice.org.uk/DT/4). Specifically, inclusion criteria are a Nottingham Prognostic Index (NPI) $>3.4$ (calculated from primary lesion size, lymph node involvement and tumour grade (Galea et al., 1992)), ER positivity, and negativity for both lymph node involvement and HER2 status.

We feel that there is value in investigating means to identify a prognostic fingerprint of wider applicability. From a computational perspective, biomarker discovery can be modelled as a feature 
selection problem that aims to classify disease into high and low risk groups according to the expression characteristics of a minimal set of discriminating genes assayed at presentation. However, most traditional feature selection methods, such as genebased techniques that use fold-change, t-test or relative entropy criteria, or group-based methods (including sequential forward/ backward selection), tend to ignore the rich biological data created by gene expression studies. Functional enrichment (i.e. the selection of genes with a known disease association) rather than simple statistical filtering methods have been used to inform cancer stratification. (Guo et al., 2005; Khunlertgit and Yoon, 2013; Wang and Chen, 2011) made use of knowledge curated in biological pathway databases (particularly the Kyoto Encyclopedia of Genes and Genomes, KEGG; www.genome.jp/kegg) or a structured biological language (notably gene ontologies described by the GO consortium; www.geneontology.org) to reduce the dimensionality of the genetic search space and increase the biological relevance of potential biomarkers identified. Previously, we described an enhancement to this pipeline, in which an initial step to identify pathways perturbed in a disease state was followed by a round of gene network analysis to further enrich for genes whose expression correlated with disease outcome (Ibrahim et al., 2012). We found that this improved disease stratification in a series of publicly-available retrospective datasets.

However, other work has cautioned that great care must be taken when data from prognostic signatures are used in clinical decision-making (Venet et al., 2011). Therefore, we sought to test the performance of a signature created using our approach to other biomarker selection methods, and to the prognostic power of the genes constituting Oncotype DX in a population of intermediate risk disease for whom additional prognostic information is particularly valuable (specifically ER positive, lymph node negative disease). Moreover, we went on to confirm the informative power of our signature relative to those of random signatures.

\section{ONCOTYPE DX BREAST CANCER GENE SET}

Oncotype DX is an RT-PCR based assay that measures the expression of 16 cancer-associated genes (as well as a panel of five internal controls) in a sample of RNA prepared from a primary tumour biopsy, returning a Recurrence Score ranging from 0 (low risk) to 100 (high risk; (Paik et al., 2004). The constituent genes are grouped by function (Figure 1 ), and were selected using a rational heuristic. We used the expression profiles of these genes in the various datasets to estimate the prognostic power of Oncotype DX.

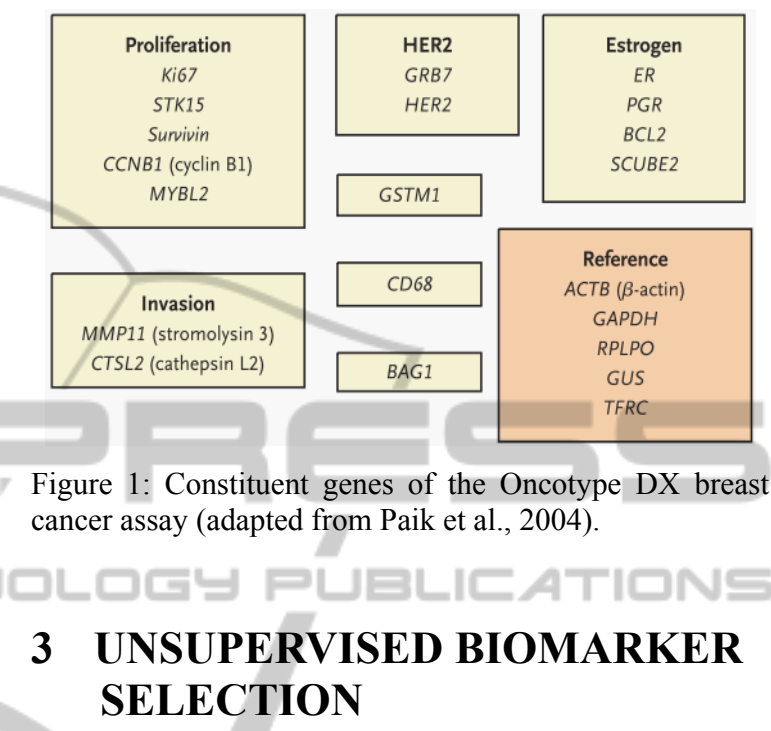

\subsection{Datasets}

Van Vliet et al, (Van Vliet et al., 2008) described a group of primary breast cancer microarray datasets that were all created using an Affymetrix U133A array platform, useful in the systematic interrogation of genetic information for prognostic insights. These data are freely available from the Gene Expression Omnibus (GEO, www.ncbi.nlm.nih.gov/geo/), with accessions GSE7390 (Desmedt et al., 2007) and GSE2990 (Loi et al., 2007), and from Array Express (www.ebi.ac.uk/arrayexpress) with the accession ETABM-158 (Chin et al., 2006). Relevant patient data (including tumour size and time of follow up) are also available from these sources. Gene expression data were normalised across all datasets using Relative Log Expression (RLE) and the Normalized Unscaled Standard Errors (NUSE) methods. In total, we were able to identify 154 samples from individuals who received no adjuvant therapy. Samples were split in to two classes according to disease outcome: class 1 (disease recurrence, 71 samples in total) and class 2 (no recurrence, 83 samples in total). Transcripts were included in biological enrichment analysis if they were differentially expressed between classes according to the following criteria: fold change $>=1.5$ and $p$ - 
value $<0.05$ (by t-test). We used median rather than mean expression values to mitigate the contribution of outliers.

\subsection{Biomarker Selection}

i. Data from individual microarrays were randomly split into training and testing sets, each set with an equal representation of disease subtypes. Specifically, the training set contained 78 samples (of which 42 had disease recurrence and 36 had no recurrence) and the testing set contained 76 samples (of which 41 had disease recurrence and 35 had no recurrence).

ii. Expression data from training sets were subjected to enrichment using pathways imported from the open-access KEGG database (www.genome.jp/kegg/). Over-representation of members of any of the 108 signalling pathways maintained in KEGG were determined by z-score as previously described (Ibrahim et al., 2012). Ranking pathways by descending score readily allowed identification of those most impacted with disease state.

iii. A gene list $p$ was created from members of $k$ high-scoring pathways whose expression could be detected on the arrays (but may not necessarily have showed a change in expression between disease states). We evaluated the performance of signatures derived from increasing values of $k$.

iv. At this stage, two methods were evaluated. To model existing pathway enrichment methods, genes were ranked by fold change, and the classification accuracy of increasing numbers of genes investigated according to stage vi). We termed this method PE_DEGs (for Pathway Enrichment and Differentially Expressed Genes). Alternatively, we proceeded to step v) in order to evaluate an improvement in performance resulting from an additional biological enrichment step. We termed this second method PEGNA for Pathway Enrichment and Gene Network Analysis.

v. The gene list $p$ was fed in to the GXNA network analysis tool (Nacu et al., 2007), to generate a user-specified number of networks. Constituent genes from networks of increasing size and number were passed to the next stage.

vi. A minimal biomarker set was identified by calculating classification accuracies using increasing numbers of genes from the filtered group, starting with five genes. The ability of the signature to separate high and low risk groups was evaluated on the testing dataset using a Support Vector Machine (SVM) classifier (Cortes and Vapnik, 1995) and a K-fold crossvalidation testing strategy (Efron and Tibshirani, 1995). If the GXNA enrichment step was used, this step was repeated for different sizes of gene network to identify the network that yielded the most informative genes.

All analysis was implemented in MATLAB 7.9.0 (Mathworks, Cambridge, UK).

\section{RESULTS}

\subsection{Prognostic Signature Identification}

We created a list of the top 10 KEGG pathways impacted when samples from relapsing and nonrelapsing individuals in the training set were compared (Table 1). All genes constituting these pathways were then fed into GXNA if they were expressed on the arrays, irrespective of any change with disease subtype.

Table 1: Top ten KEGG pathways impacted in relapsing breast cancer ranked by z-score.

\begin{tabular}{|c|c|c|}
\hline & Pathway & z-score \\
\hline 1 & Dorso-ventral axis formation & 3.31 \\
\hline 2 & Calcium signaling pathway & 2.82 \\
\hline 3 & Bladder cancer & 2.03 \\
\hline 4 & Chemokine signaling pathway & 1.55 \\
\hline 5 & Endocytosis & 1.51 \\
\hline 6 & Cardiac muscle contraction & 1.46 \\
\hline 7 & Bacterial invasion of epithelial cells & 1.46 \\
\hline 8 & Focal adhesion & 1.38 \\
\hline 9 & Regulation of actin cytoskeleton & 1.34 \\
\hline 10 & VEGF signaling pathway & 1.33 \\
\hline
\end{tabular}

The testing set was used to build the SVM classifier and to evaluate the performance of the signature. Seventy-five of the 76 testing samples were used to train the SVM classifier, which was then tested on the remaining sample. This step was repeated 76 times, with a different sample used each time to test the classifier.

The accuracy achieved when the trained classifier was used to separate samples into highand low-risk groups based on gene expression profiles is shown in Figure 2. While the use of 20 and 24 genes isolated from the top 10 pathways gave the same accuracy $(73.7 \%)$, we selected latter group due to the high sensitivity achieved by this group 
(74.3\%) compared to the 20 gene signature $(68.6 \%$, Figure 3).

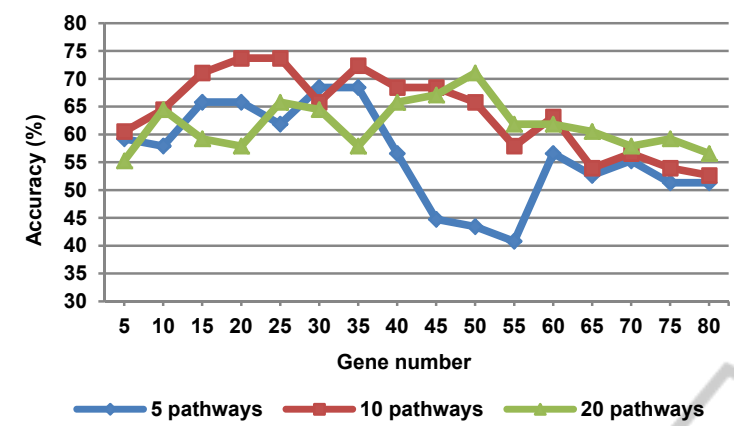

Figure 2: Evaluation of increasing gene numbers selected from the 5, 10 and 20 highest scoring KEGG pathways.

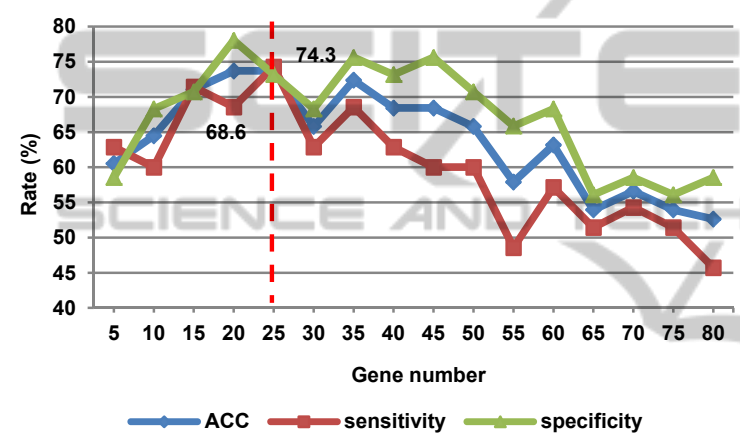

Figure 3: Accuracy (ACC), sensitivity and specificity achieved with increasing numbers of genes derived from the 10 highest scoring KEGG pathways.

A comparison between PEGNA and PE_DEGs confirmed the effectiveness of combining biological enrichment methods in obtaining a more informative signature (Figure 4). PEGNA achieved a maximum accuracy of $73.7 \%$ with just 24 genes, whereas PE_DEGs achieved a maximum $56.6 \%$, and this required 40 genes. A single round of GXNA enrichment alone (with no prior pathway enrichment) did not match the performance of the combined method, possibly as this technique tends to find sub-optimal solutions on larger datasets (data not shown).

\subsection{Performance}

While accuracy is a useful headline indicator of the ability of a classifier to identify those at risk of relapse, more meaningful measures include sensitivity (individuals with recurrent disease that is accurately predicted by the signature) and specificity (accurate prediction of individuals in which disease won't reoccur). Another useful measure is an

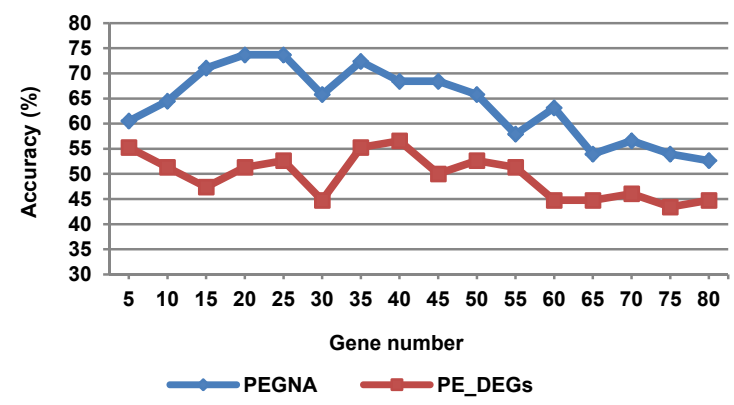

Figure 4: Accuracy rates achieved by PE_DEGs and PEGNA with increasing numbers of genes from the top 10 scoring pathways.

indication of the number of patients who would have been over- or under-treated, had therapeutic regimes been tailored to a predicted disease course. Results of this analysis are shown in Table 2.

Table 2: A comparison of the performance of a novel 24 gene PEGNA-derived signature with the 16 genes constituting Oncotype DX in classifying a retrospective untreated group of $\mathrm{ER}+, \mathrm{LN}$ - breast cancer.

\begin{tabular}{|c|c|c|}
\hline & Oncotype DX & PEGNA \\
\hline Accuracy & $59 \%$ & $73.7 \%$ \\
\hline Sensitivity & $48.6 \%$ & $74 \%$ \\
\hline Specificity & $68 \%$ & $73 \%$ \\
\hline True positives & 17 & 26 \\
\hline $\begin{array}{c}\text { False positives } \\
\text { (over-treated) }\end{array}$ & 13 & 11 \\
\hline $\begin{array}{c}\text { True negatives } \\
\text { False negative }\end{array}$ & 28 & 30 \\
(Under-treated) & 18 & 9 \\
\hline
\end{tabular}

\subsection{Statistical Evaluation of Prognostic Signature Performance}

To evaluate the statistical significance of the accuracy of the genes constituting the Oncotype DX panel and those identified by PEGNA, we determined the probability of achieving similar or higher classification accuracies by chance. A group of 24 genes was randomly selected from the list of all expressed microarray genes (of which there were 14368 in total) from the microarray dataset used to define the original signature. This random signature was then used to classify the testing samples and an 
accuracy value was determined. This process was repeated 1000 times, each with a distinct random signature from which probability was estimated in two different ways.

Firstly, the number of random signatures that were at least as accurate as our bespoke signature was calculated. We found that only two random signatures out of the 1000 performed as well as our signature. A p-value for the accuracy of our signature $\left(\mathrm{ACC}_{\mathrm{PEGNA}}\right)$ was then calculated using Equation 1, and found to be 0.002 (i.e. 2/1000). In the same way, the p-value associated with the accuracy achieved by the genes constituting the Oncotype DX signature was calculated to be 0.126 (i.e. 126/1000).

$$
P\left(A C C_{P E G N A}\right)=\frac{\sum_{j=1}^{n} I\left(A C C_{\text {Rand }} \geq A C C_{P E G N A}\right)}{n}
$$

Where $\mathrm{I}(\mathrm{X})$ is the identity function that returns 1 if $\mathrm{X}$ is true and 0 if false, and $\mathrm{P}\left(\mathrm{ACC}_{\mathrm{PEGNA}}\right)$ is the probability of achieving the accuracy of PEGNA signature by chance.

Secondly, we modelled the classification accuracy distribution of the random signatures according to the standard normal distribution. Figure 5 confirms that the histogram of the 1000 classification accuracies achieved by the random signatures is normally distributed. By calculating the mean and the standard deviation of classification accuracies, one can work out a z-score for the accuracy of the PEGNA-derived signature using the formula given by Equation 2. By using the standard normal distribution table, a p-value for an $\mathrm{ACC}_{\mathrm{PEGNA}}$ value of $73.7 \%$ was estimated to be 0.0024 . In contrast, the p-value associated with achieving an accuracy value of 59\% using the Oncotype DX signature by chance was 0.117 using the same method.

$$
Z_{P E G N A}=\frac{A C C_{P E G N A}-A C C_{\text {mean }}}{A C C_{\sigma}}
$$

Where $\mathrm{ACC}_{\text {mean }}$ and $\mathrm{ACC}_{\sigma}$ are the mean and the standard deviation of the accuracy distribution of the random signatures.

\section{CONCLUSIONS}

The increasingly multidisciplinary management of breast cancer has led to a significant improvement in survival outcomes. One reason for this improvement is the use of research into improved prognostication and prediction of treatment response. For example, the advent of microarray

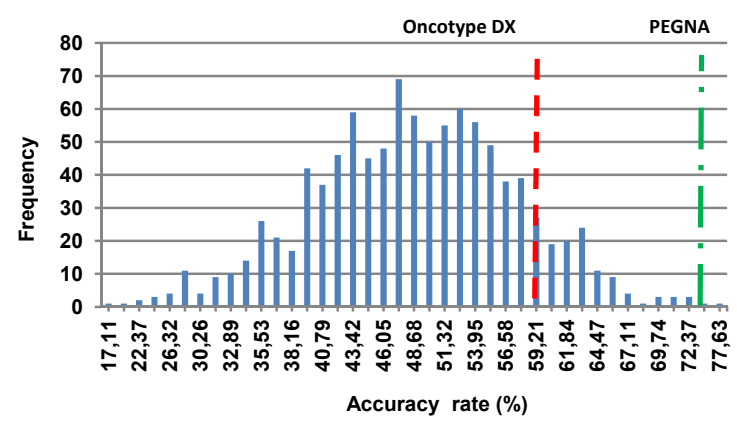

Figure 5: Distribution of accuracy values achieved by 1000 random 24 gene signatures. Performance of both Oncotype DX and PEGNA-derived signatures are indicated.

technology has led to the identification of specific molecular signatures that have enhanced the accuracy of traditional tumour prognostic factors, for example lymph node status. However, despite these improvements, there remains a degree of uncertainty in tailoring adjuvant treatment to patients in certain prognostic groups. For example, the Intermediate Risk of Recurrence patient category (based on the calculated NPI and Oncotype DX analysis) is a difficult group for which to plan treatment. While we tested our algorithm on a broad selection of ER+, lymph node negative disease, we also attempted to evaluate the performance of molecular signatures on a sub group of disease meeting the NICE guidelines of NPI $>3.4$ and HER2 negativity, but the number of samples that met these criteria represented only a very small subgroup of intermediate risk disease. It is also important to note that we did not use the Oncotype DX assay per se, rather we trained our classier to discriminate between disease groups based on the expression of its constituent genes identified in a larger microarray dataset, which will most likely have implications for performance.

The experiments presented in this paper provide a proof-of-concept for the potential clinical utility of genetic signatures derived from computational methods, and we feel that our approach will enhance prognostic and predictive value. We intend to validate our results by conducting further studies on the correlation between tumour gene expression characteristics and patient outcomes in a clinical setting.

\section{ACKNOWLEDGEMENTS}

We are grateful to Professor Karol Sikora for helpful comments on the manuscript. 


\section{REFERENCES}

Chin, K., DeVries, S., Fridlyand, J., Spellman, P.T., Roydasgupta, R., Kuo, W.-L., Lapuk, A., Neve, R.M., Qian, Z., Ryder, T., Chen, F., Feiler, H., Tokuyasu, T., Kingsley, C., Dairkee, S., Meng, Z., Chew, K., Pinkel, D., Jain, A., Ljung, B. M., Esserman, L., Albertson, D. G., Waldman, F. M., Gray, J. W., 2006. Genomic and transcriptional aberrations linked to breast cancer pathophysiologies. Cancer Cell 10, 529-541.

Cortes, C., Vapnik, V., 1995. Support-vector networks. Machine learning 20, 273-297.

Desmedt, C., Piette, F., Loi, S., Wang, Y., Lallemand, F., Haibe-Kains, B., Viale, G., Delorenzi, M., Zhang, Y., D' Assignies, M. S., Bergh, J., Lidereau, R., Ellis, P., Harris, A. L., Klijn, J. G. M., Foekens, J. A., Cardoso, F., Piccart, M. J., Buyse, M., Sotiriou, C., On behalf of the TRANSBIG Consortium, 2007. Strong time dependence of the 76-gene prognostic signature for node-negative breast cancer patients in the TRANSBIG multicenter independent validation series. Clinical Cancer Research 13, 3207-3214.

Efron, B., Tibshirani, R., 1995. Cross-validation and the bootstrap: Estimating the error rate of a prediction rule. Division of Biostatistics.

Galea, M. H., Balmey, R. W., Elston, C. E., Ellis, I. O., 1992. The Nottingham Prognostic Index in primary breast cancer. Breast cancer research and treatment 22, 207-219.

Guo, Z., Zhang, T., Li, X., Wang, Q., Xu, J., Yu, H., Zhu, J., Wang, H., Wang, C., Topol, E., others, 2005. Towards precise classification of cancers based on robust gene functional expression profiles. $B M C$ Bioinformatics 6, 58.

Ibrahim, M., Jassim, S., Cawthorne, M.A., Langlands, K., 2012. Integrating pathway enrichment and gene network analysis provides accurate disease classification. The International Conference on Bioinformatics Models, Methods and Algorithms. 156163.

Khunlertgit, N., Yoon, B.-J., 2013. Identification of robust pathway markers for cancer through rank-based pathway activity inference. Advances in Bioinformatics 2013, 1-8.

Loi, S., Haibe-Kains, B., Desmedt, C., Lallemand, F., Tutt, A. M., Gillet, C., Ellis, P., Harris, A., Bergh, J., Foekens, J. A., Klijn, J. G. M., Larsimont, D., Buyse, M., Bontempi, G., Delorenzi, M., Piccart, M. J., Sotiriou, C., 2007. Definition of clinically distinct molecular subtypes in estrogen receptor-positive breast carcinomas through genomic grade. Journal of Clinical Oncology 25, 1239-1246.

Nacu, Ş., Critchley-Thorne, R., Lee, P., Holmes, S., 2007. Gene expression network analysis and applications to immunology. Bioinformatics 23, 850-858.

Paik, S., Shak, S., Tang, G., Kim, C., Baker, J., Cronin, M., Baehner, F. L., Walker, M. G., Watson, D., Park, T., 2004. A multigene assay to predict recurrence of tamoxifen-treated, node-negative breast cancer. New England Journal of Medicine 351, 2817-2826.
Van Vliet, M. H., Reyal, F., Horlings, H. M., Van de Vijver, M. J., Reinders, M. J., Wessels, L. F., 2008. Pooling breast cancer datasets has a synergetic effect on classification performance and improves signature stability. BMC Genomics 9, 375.

Van't Veer, L. J., Dai, H., Van De Vijver, M. J., He, Y. D., Hart, A. A. M., Mao, M., Peterse, H. L., Van Der Kooy, K., Marton, M. J., Witteveen, A. T., others, 2002. Gene expression profiling predicts clinical outcome of breast cancer. Nature 415, 530-536.

Venet, D., Dumont, J. E., Detours, V., 2011. Most random gene expression signatures are significantly associated with breast cancer outcome. PLoS Computational Biology 7, e1002240.

Wang, Y. C., Chen, B. S., 2011. A network-based biomarker approach for molecular investigation and diagnosis of lung cancer. BMC medical genomics 4, 2.

Wesolowski, R., 2011. Gene expression profiling: changing face of breast cancer classification and management. Gene expression 15, 105-115.

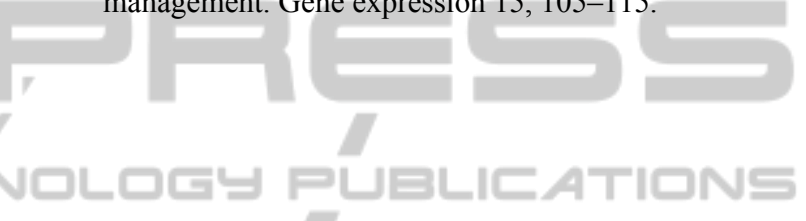

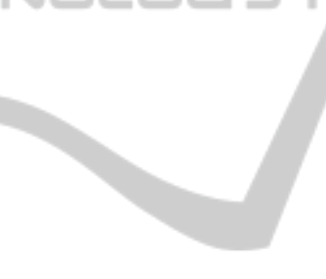

